\title{
CASCADE DEBRIS OVERLAP MECHANISM OF $<100>$ DISLOCATION LOOP FORMATION IN Fe AND FeCr
}

Granberg, F.

2017

Granberg , F , Byggmästar, J , Sand , A E \& Nordlund, K 2017 , ' CASCADE DEBRIS OVERLAP MECHANISM OF $<100>$ DISLOCATION LOOP FORMATION IN Fe AND FeCr ' , Europhysics Letters , vol. 119 , 56003 . https://doi.org/10.1209/0295-5075/119/56003

http://hdl.handle.net/10138/308771

https://doi.org/10.1209/0295-5075/119/56003

cc_by_nc_nd

acceptedVersion

Downloaded from Helda, University of Helsinki institutional repository.

This is an electronic reprint of the original article.

This reprint may differ from the original in pagination and typographic detail.

Please cite the original version. 
See discussions, stats, and author profiles for this publication at: https://www.researchgate.net/publication/321122001

\section{Cascade debris overlap mechanism of $\square 100 \square$ dislocation loop formation in Fe and $\mathrm{FeCr}$}

Article in EPL (Europhysics Letters) · September 2017

DOI: 10.1209/0295-5075/119/56003

CITATIONS

8

4 authors:

Fredric Granberg

University of Helsink

34 PUBLICATIONS 335 CITATIONS

SEE PROFILE

Andrea E Sand

University of Helsinki

43 PUBLICATIONS 424 CITATIONS

SEE PROFILE

Some of the authors of this publication are also working on these related projects:

Project Ion beam theory View project

Project $\quad$ Extreme Irradiation of Materials: Fusion Reactor Conditions in ITER and Beyond (EXMAT) View project
READS

122

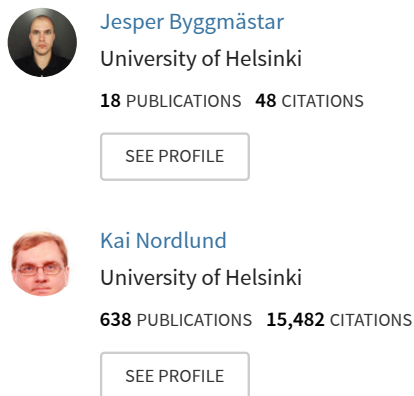


Preprint of article published in Europhysics Letters 119 (2017) 56003, doi: 10.1209/0295-5075/119/56003

\title{
Cascade debris overlap mechanism of $\langle\mathbf{1 0 0}\rangle$ dislocation loop formation in $\mathrm{Fe}$ and $\mathrm{FeCr}$
}

\author{
F. Granberg ${ }^{1}$, J. Byggmästar ${ }^{1}$, A. E. Sand ${ }^{1,2}$ and K. Nordlund ${ }^{1}$ \\ ${ }^{1}$ Department of Physics, P.O. Box 43, \\ FI-00014 University of Helsinki, Finland and \\ ${ }^{2}$ CCFE, Culham Science Centre, Abingdon, Oxon, OX14 3DB, UK*
}

\begin{abstract}
Two types of dislocation loops are observed in irradiated $\alpha$-Fe, the $1 / 2\langle 111\rangle$ loop and the $\langle 100\rangle$ loop. Atomistic simulations consistently predict that only the energetically more favourable $1 / 2\langle 111\rangle$ loops are formed directly in cascades, leaving the formation mechanism of $\langle 100\rangle$ loops an unsolved question. We show how $\langle 100\rangle$ loops can be formed when cascades overlap with random pre-existing primary radiation damage in Fe and FeCr. This indicates that there are no specific constraints involved in the formation of $\langle 100\rangle$ loops, and can explain their common occurrence.
\end{abstract}




\section{INTRODUCTION}

Iron-based and iron-chromium-based alloys are the most widely used construction metals. They are also the materials used in nuclear power plant vessels, and hence knowing their irradiation response is crucial. One important property of the structural materials in power plants is their mechanical behaviour, and the possible detrimental changes that can be induced by irradiation. In metals, the mechanical properties are mainly determined by the existence and movement of dislocations ${ }^{5}$. The migrational properties of nano-sized dislocation loops, formed as products of irradiation, are strongly dependent on their Burgers vector, and therefore knowing what type of dislocation loops are formed is crucial for predicting the subsequent microstructural evolution of the material. While $1 / 2\langle 111\rangle$ loops can easily migrate and be absorbed by defect sinks like grain boundaries and surfaces, $\langle 100\rangle$ loops are highly immobile, and can themselves act as defect sinks in the material. Understanding the formation mechanisms of the $\langle 100\rangle$ loops is therefore important.

It has been known since experiments in the 1960s, that both $1 / 2\langle 111\rangle$ and $\langle 100\rangle$ dislocation loops are formed as a result of irradiation in $\mathrm{Fe}^{16,34}$, although the $1 / 2\langle 111\rangle$ loops are energetically more stable ${ }^{32}$. This is in contrast to other BCC metals such as V and Mo, where $\langle 100\rangle$ loops are rarely seen ${ }^{23,24}$. Until recently, it was believed that such loops do not form in $\mathrm{W}$ either, but they have now been shown to form in individual massive collision

cascades $^{25,35}$. However, because of the low subcascade splitting energy in $\mathrm{Fe}^{28}$, such massive cascade events rarely occur in neutron- or self-ion-irradiated Fe. Hence, even though the $\langle 100\rangle$ dislocation loops are regularly seen experimentally, the underlying mechanisms of formation in Fe are not yet fully known. In this Letter, we show how homogeneous prolonged irradiation of Fe and Fe-based materials leads spontaneously to the formation of $\langle 100\rangle$ loops.

A key aspect of radiation damage in Fe is that TEM-visible defects, in particular those large enough to allow determination of their Burgers vector, form only after a time, when the radiation dose (as commonly measured by displacements-per-atom, dpa ${ }^{20}$ ) has reached a certain level. Thus, the observed $\langle 100\rangle$ loops may be products of the evolved, initially sub-visible, defects. Experimentally, $\langle 100\rangle$ loops have been seen to form from a spontaneous change in the Burgers vector from a $1 / 2\langle 111\rangle$ loop to a $\langle 100\rangle$ loop upon heating ${ }^{2}$. This spontaneous transformation was later attributed to anisotropic elasticity effects in iron ${ }^{6}$, an effect which becomes increasingly important at higher temperatures. 
The formation of $\langle 100\rangle$ interstitial-type dislocation loops in molecular dynamics simulations of cascades in Fe has been reported only for very energetically dense cascades ${ }^{4}$, in events similar to those responsible for the formation of $\langle 100\rangle$ loops in W. Such dense cascades in Fe are mainly the result of primary recoil atoms which are much more massive than the Fe atom itself. This indicates that direct nucleation of $\langle 100\rangle$ loops from individual cascades in neutron or self-ion irradiation in Fe is unlikely. Formation of $\langle 100\rangle$ loops was also reported for cascades on existing $1 / 2\langle 111\rangle$ loops, where the initial loops were dissolved by the cascades and in some cases reformed as a $\langle 100\rangle$ loop $^{31}$. One instance of a vacancy-type $\langle 100\rangle$ dislocation loop forming in bulk Fe has been reported ${ }^{26}$. However, this single event occurred once in 100 cascades, and has not been observed since, nor with other interatomic potentials, despite a massive body of cascade simulations in Fe reported in the literature to date. A similar cascade collapse event has also been observed in $\mathrm{W}^{25}$, but whether these occurrences are physical, or should be attributed to flukes in the particular interatomic potentials, is still unclear. In near-surface cascades, however, it is found that large vacancy clusters tend to form much more readily, and in this case also cascade collapse into vacancy-type loops is more likely to occur ${ }^{1}$.

Several other mechanisms have been proposed, based on atomistic simulations, for the formation of $\langle 100\rangle$ interstitial loops from the evolution of existing defect structures ${ }^{3,14,33,37}$. These mechanisms rely either on the combination and transformation of $1 / 2\langle 111\rangle \operatorname{loops}^{3,14,33}$ or on the formation of large C15 interstitial clusters in irradiated Fe, experimentally not yet verified ${ }^{37}$. In all of the previous studies, the loop formation was the result of some artificially made defect configuration, positioned in a manner favouring $\langle 100\rangle$ loop formation. In this Letter, we show how homogeneous prolonged irradiation leads spontaneously to the formation of $\langle 100\rangle$ loops in Fe and Fe-based materials. This alternative formation mechanism, based on the overlap of cascades with pre-existing cascade damage, is not in contradiction to previous mechanisms, but does not require a predefined starting point. This mechanism is in line with the fact that observed $\langle 100\rangle$ loops in Fe are the result of irradiation to a fairly high dpa ${ }^{34}$, where overlap effects can be significant. We study overlapping cascades both in bulk systems and in systems with a close by surface, the latter to include a permanent defect sink for mobile defects. To simulate the effects of high doses of irradiation, we run massively overlapping cascades, where debris of multiple previous cascades can be found. 


\section{METHODOLOGY}

We use molecular dynamics (MD) to simulate the defect production in Fe and FeCr-alloys. To obtain doses comparable to experiments, we performed 2000 overlapping cascades with a constant primary knock-on atom (PKA) energy of $5 \mathrm{keV}$, reaching a dose corresponding to 0.12 dpa. Previous investigations utilizing a similar method have shown good agreement between simulations and experimentally obtained results ${ }^{36}$. The starting point of the simulations was a perfect lattice of BCC Fe with different chromium content (from pure Fe to $\left.\mathrm{Fe}_{80} \mathrm{Cr}_{20}\right)$. Periodic boundary conditions were applied in all directions to simulate bulk material, while systems with a defect sink were investigated with an open surface on top and a fixed bottom in the $z$-direction, and periodic boundaries in the other directions. The simulations were carried out with the MD code PARCAS ${ }^{9,19}$ and the interatomic potential for FeCr by Olsson et al. ${ }^{21}$. The FeCr potential uses the Ackland-Mendelev EAM-type potential for the pure Fe part ${ }^{17}$. To test for dependencies on interatomic potential, three sets of massively overlapping cascades in $\mathrm{Fe}$ and $\mathrm{FeCr}$ systems were also performed with a Tersoff-type potential ${ }^{10}$. To deal with the high dose rate associated with the overlapping cascades in MD, we also carried out several series of individual secondary cascades at 0 K (40 simulations in each series). A slight rise in temperature, of a few hundred degrees,

has negligible effect on the primary damage formed in single cascades ${ }^{18,27}$. For this reason, we study single cascades simulated at $0 \mathrm{~K}$ in order to fully isolate the cascade dynamics from thermally induced evolution. On the other hand, the cooling rate of the heat spike is affected by the ambient temperature, becoming slower at raised temperatures, thus the cascade-induced migration and transformations of defects in the simulation cell will be sensitive also to the ambient temperature. Hence, we have performed the massively overlapping cascades at $300 \mathrm{~K}$ in order to correspond to room temperature experiments. The series of secondary cascades were conducted to investigate the statistics of the overlapping effects of cascades, on various configurations of pre-existing damage in pure Fe, using the AcklandMendelev potential ${ }^{17}$. For these simulations, we used a higher PKA energy (50 keV) to cover the overlapping effects and possible dislocation nucleations in larger areas. These simulations were repeated with the interatomic potential by Dudarev et al. ${ }^{7}$, to confirm that our findings were not due to a specific property of the potential. Dislocations and their Burgers vectors were systematically identified using the dislocation extraction algorithm (DXA) im- 
plemented in OVITO ${ }^{29,30}$. More details on the systems and simulation details can be found in the Supplementary material available online.

\section{RESULTS AND DISCUSSION}

The results show that the overlap of the disordered core of a cascade with a pre-existing defect cluster is enough to lead to the formation of an interstitial-type $\langle 100\rangle$ dislocation loop with a significant probability. Even though mainly $1 / 2\langle 111\rangle$ dislocation loops are seen in the massively overlapping cascades (see Fig. 1), there was at least one perfect $\langle 100\rangle$ loop present in $4 \%$ of the frames. The interstitial-type $\langle 100\rangle$ loops were observed in pure Fe as well as at all different chromium contents. In $20 \%$ of the $50 \mathrm{keV}$ cascade cases, the cascade yielded a perfect $\langle 100\rangle$ interstitial loop in the direct overlap of a previous dislocation network. In the massively overlapping $5 \mathrm{keV}$ cascades, $\langle 100\rangle$ loops formed at some point in all simulation cells with all interatomic potentials. The formed loops are usually around $1-2 \mathrm{~nm}$ in size. Defects of such size are regularly produced in more energetic single cascades, but require evolution of the microstructure or significant cascade overlap in the case of lower energy cascades. The pre-existing damage that we observe leading to the formation of $\langle 100\rangle$ loops is in general irregular, often consisting of a network of $1 / 2\langle 111\rangle$ and $\langle 100\rangle$ segments (see Fig. 1), or of $1 / 2\langle 111\rangle$ segments with their Burgers vector in different directions.

In some cases, we observe a large interstitial $\langle 100\rangle$ loop forming from two existing $1 / 2\langle 111\rangle$ loops, similar to the mechanism proposed in Refs. ${ }^{14},{ }^{33}$ and $^{3}$. Marian et al. proposed a mechanism with a recombination of two similarly sized $1 / 2\langle 111\rangle$ loops into a $\langle 100\rangle$ loop, and subsequent growth by absorption of smaller $1 / 2\langle 111\rangle \operatorname{loops}^{14}$. Their simulations did not, however, reproduce the formation of a complete $\langle 100\rangle$ loop. Xu et al. ${ }^{33}$ and Beland et al. ${ }^{3}$, on the other hand, showed a complete formation of $\langle 100\rangle$ loops by the interaction and transformation of two $1 / 2\langle 111\rangle$ loops in both Fe and FeCr. However, as this formation mechanism requires that the two $1 / 2\langle 111\rangle$ loops are of equal size, it is not very likely to happen in a real situation. In contrast to their results, however, we show that the transformation is mediated by the close proximity of cascade impacts, and does not require two equally sized and perfect $1 / 2\langle 111\rangle$ loops, but rather acts on two irregular loops. In Fig. 2, we show how two $1 / 2\langle 111\rangle$ loops combine to a mixed dislocation network, as a result of one overlapping cascade (Figs. 2a and b). It then remains in the same configuration for over 250 cascades, 


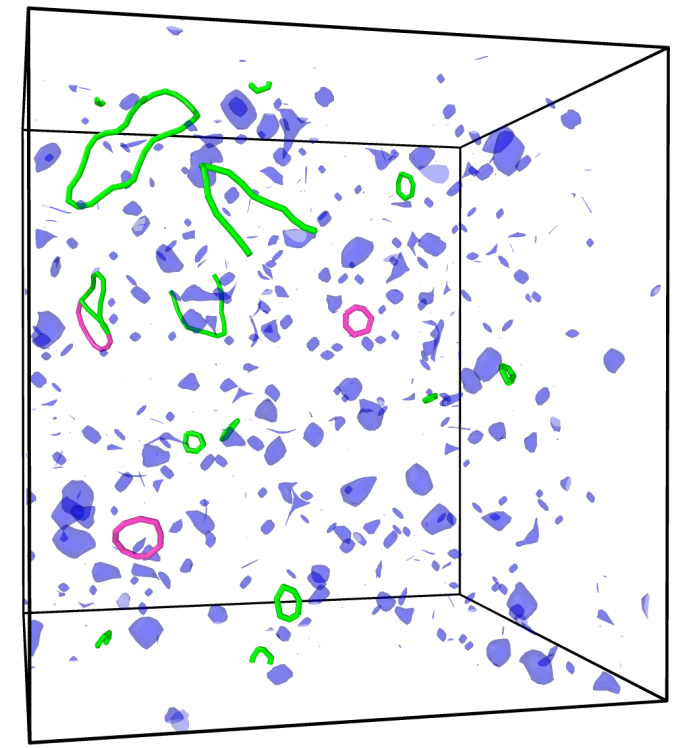

FIG. 1: Defects obtained from the DXA analysis in the simulation cell after $\sim 1000$ cascades. The dark (online: green coloured) lines represent $1 / 2\langle 111\rangle$-segments and the light (online: light violet) lines represent the $\langle 100\rangle$-segments. The light gray (online: blue) mesh represents a defective area, that cannot be identified as a dislocation.

with some small transformations. In Fig. 2c, the configuration before the following overlapping cascade is shown; in this case the cascade partly envelopes the dislocation structure, which initiates the transformation seen in Figs. 2d to f. A complete movie of the partial destruction and transformation can be seen in the Supplementary material. A similar mechanism, where two different sized $1 / 2\langle 111\rangle$ loops combine, and eventually transform into a $\langle 100\rangle$ loop, is seen in several of the simulation runs.

We also observe that some of the mixed interstitial $1 / 2\langle 111\rangle-\langle 100\rangle$ loops, generated directly in the cascades, can be transformed into a complete $\langle 100\rangle$ loop. This happens via a combination of (i) destruction of parts of the $1 / 2\langle 111\rangle$ segments by the cascade and (ii) absorption of the $1 / 2\langle 111\rangle$ part into a $\langle 100\rangle$ loop, similar to that presented by Marian et al. in Ref. 14. They reported that a $\langle 100\rangle$ loop can absorb small $1 / 2\langle 111\rangle$ loops and grow, but they relied on that the initial $\langle 100\rangle$ was created by combination of two $1 / 2\langle 111\rangle$ loops, which they did not observe. One occurrence of a cascade induced transformation of a mixed loop is illustrated in Fig. 3. The initial mixed dislocation network, shown in Fig. 3a, contained 47 interstitials and was produced randomly from two overlapping cascades. This was part of the initial damage state for a series of 40 individual $50 \mathrm{keV}$ cascade simulations. A subse- 


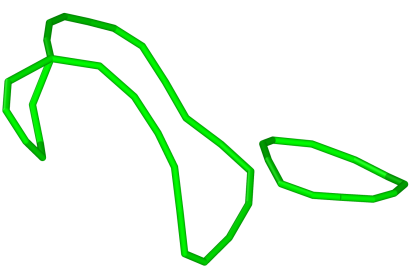

(a) Before cascade 1026

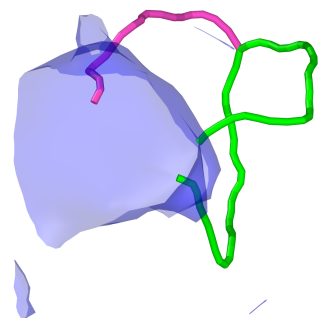

(d) During cooldown

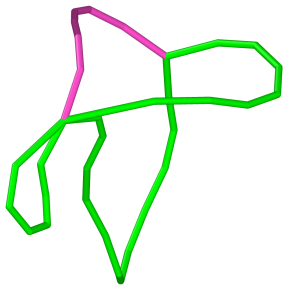

(b) After cascade 1026

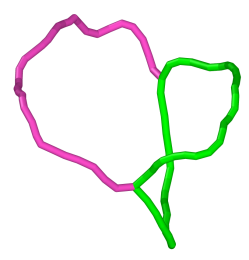

(e) During cooldown

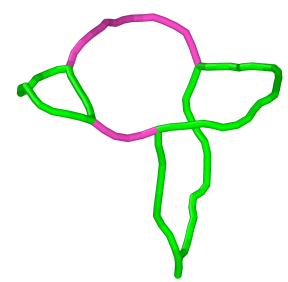

(c) Before cascade 1288

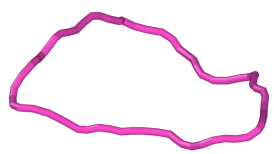

(f) After cascade 1288

FIG. 2: Combination of two interstitial $1 / 2\langle 111\rangle$ loops and transformation into a perfect 3 $\mathrm{nm}\langle 100\rangle$ loop. The colouring is the same as for Fig. 1

quent overlapping cascade produced a $\langle 100\rangle$ loop in 2 cases out of 10 , while a $1 / 2\langle 111\rangle$ loop was produced in the remaining 8 instances of significant overlap. In the other 30 simulations of that particular series, the cascade did not directly overlap the mixed dislocation network, and correspondingly little or no change in the structure was observed, thus indicating that direct overlap of the disordered cascade region with the defect is necessary for this transformation mechanism to operate. When a transformation occurs, the mixed loop is partially or completely destroyed in the molten cascade region generated by the secondary cascade (Fig 3b), and after cooling regenerates, e.g. in the form seen in Fig. 3c. In the illustrated case, as the cascades cools further, the new dislocation network eventually transforms into a perfect $\langle 100\rangle$ loop in a sequence of two $1 / 2\langle 111\rangle$ segments combining to form $\langle 100\rangle$ segments. In Fig. 3c and d, the dislocation segments terminate in the still molten cascade region. In Fig. 3e, the loop is fully formed, and consists of two $1 / 2\langle 111\rangle$ loops connected by two $\langle 100\rangle$ segments. In Fig. 3f, an additional $\langle 100\rangle$ segment is formed through the reaction $1 / 2[\overline{1} \overline{1} \overline{1}]+1 / 2[11 \overline{1}]=[00 \overline{1}]$. Finally in Fig. $3 \mathrm{~g}$, the $\langle 100\rangle$ loop is completed via a transformation of the remaining $1 / 2\langle 111\rangle$ segments combining as $1 / 2[1 \overline{1} \overline{1}]+1 / 2[\overline{1} 1 \overline{1}]=[00 \overline{1}]$. The 
created $\langle 100\rangle$ loop contains about 30 interstitials, which is $35 \%$ fewer than in the initial dislocation network. The occurrence where the $1 / 2\langle 111\rangle$ segment of a mixed loop is destroyed by the cascade and transformed into a $\langle 100\rangle$ loop is seen in Fig. 4. In this figure, the interstitials in a $\langle 111\rangle$ orientation are reoriented into the $\langle 100\rangle$ orientation by the energetic cascade. The large (online: red) dots are the actual interstitial atoms, and the small (online: blue) dots the positions according to the Wigner-Seitz analysis. The light mesh is the defective volume according to OVITO's dislocation analysis. A complete movie of this transformation can be seen in the Supplementary material. The number of interstitials associated with the defect is reduced during the cascade, leaving fewer point defects in the transformed loop in Fig. 4. In all cases presented here (Figs. 2 - 4), the total length of the dislocation segments is also reduced, as is the number of interstitials in Figs. 3 and 4. Hence the transformation does not necessarily involve an increase in energy, even though the $\langle 100\rangle$-dislocation loops have a higher energy per unit length/size of dislocation ${ }^{13}$.

These transformations are stochastic in nature, with some of the mixed loops transforming into $\langle 100\rangle$ loops and some into $1 / 2\langle 111\rangle$ loops. We also observed direct formation of small $\langle 100\rangle$ loops in locations where an overlapping cascade fully encompassed a small pre-existing interstitial cluster or loop, resulting in a locally dense region within the heat spike. This was observed in all samples, both bulk and bulk with a surface as well as at all investigated chromium concentrations. The addition of chromium did not affect the production of $\langle 100\rangle$ loops, as the loops were seen at least as often in FeCr as in pure Fe. Hence, even low energy cascades can introduce $\langle 100\rangle$ loops to the sample, when previous cascade debris exists. In the study of individual secondary cascades, we observed that the initial debris from only one 50 $\mathrm{keV}$ cascade was enough for small $\langle 100\rangle$ loops, or $\langle 100\rangle$ dislocation segments, to be created as a result of a subsequent cascade. The formation of interstitial type $\langle 100\rangle$ loops were observed with all three interatomic potentials used here, and are therefore not a potentialspecific phenomenon, but a general feature of overlapping cascades. The cascade-induced combination and transformation of $1 / 2\langle 111\rangle$ loops into $\langle 100\rangle$ loops, and the spontaneous formation of $\langle 100\rangle$ loops in overlapping cascades, indicates that $\langle 100\rangle$ loops can form in more general conditions than those assumed in previous studies. Occasionally, vacancy-type $\langle 100\rangle$ loops are observed in these setups, but are not the subject of the current study. The presented mechanism is not in contradiction with the work of Zhang et al. that showed that growth of C15 interstitial clusters in iron can eventually lead to a transformation 
into $\langle 100\rangle$ loops at a critical $\operatorname{size}^{37}$. However, the formation and growth of the C15 Laves phase in irradiated Fe has yet to be experimentally verified. Although the C15 structure is energetically stable, its growth relies on the capture of single SIAs ${ }^{15}$, a process which is unassisted by the elastic strain fields that drive the experimentally observed growth by coalescence of dislocation loops ${ }^{8}$.

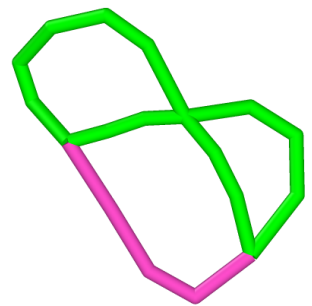

(a) 0 ps

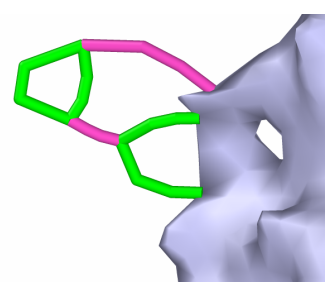

(d) $5.69 \mathrm{ps}$

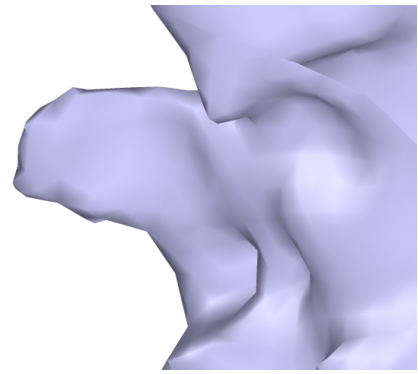

(b) $4.55 \mathrm{ps}$

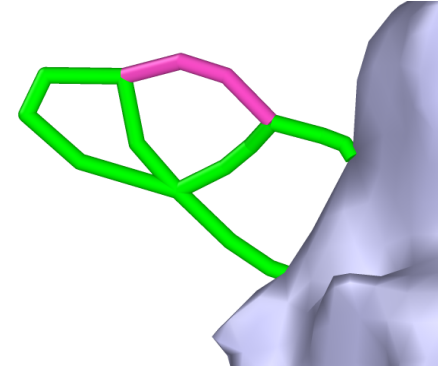

(c) $5.66 \mathrm{ps}$

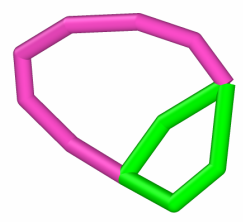

(f) $7.72 \mathrm{ps}$

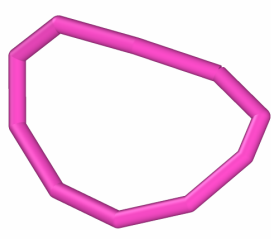

(g) $11.76 \mathrm{ps}$

FIG. 3: Cascade-induced transformation of a mixed interstitial loop into a perfect $1 \mathrm{~nm}$ $\langle 100\rangle$ loop via reactions of partial $1 / 2\langle 111\rangle$ segments. The colouring is the same as for Fig. 1

Although the overall probability of complex mixed loops transforming into perfect $\langle 100\rangle$ loops is low, $\langle 100\rangle$ loop formation is still significantly frequent when the density of defect clusters in the material becomes high. Additionally, over a longer time scale, a significant portion of the $1 / 2\langle 111\rangle$ loops are likely to migrate and be absorbed by grain boundaries and surfaces. Hence the density of the immobile $\langle 100\rangle$ loops can grow to higher fractions, comparable to those observed in experiments. In the simulations of massively overlapping cascades with an open surface, $1 / 2\langle 111\rangle$ loops are often seen to migrate and recombine at the surface, whereas the $\langle 100\rangle$ loops remain stable and immobile over a large number of subsequent cascades. While the formation mechanism of $\langle 100\rangle$ loops proposed here relies crucially on the effects of the molten cascade core reforming parts of pre-existing dislocation 


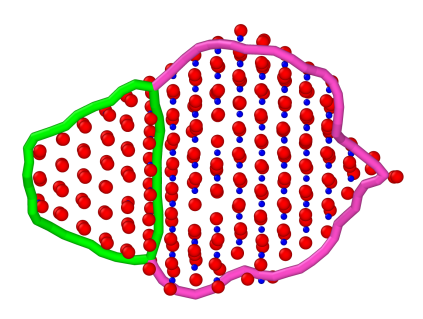

(a) 0 ps

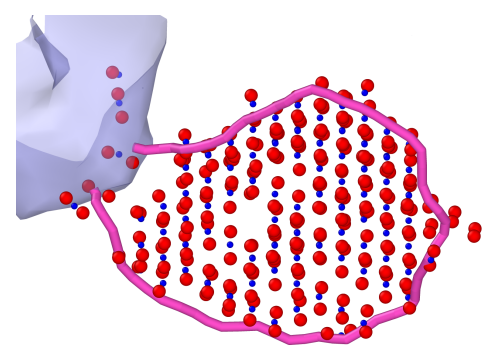

(d) $2.15 \mathrm{ps}$

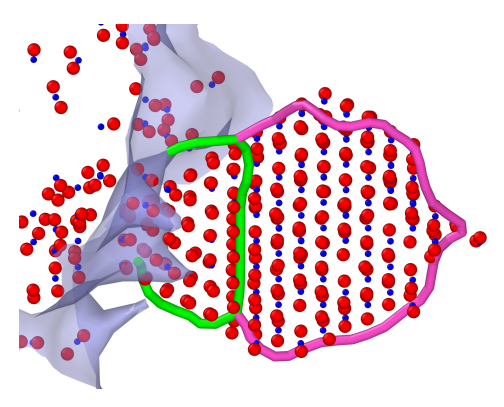

(b) $0.19 \mathrm{ps}$

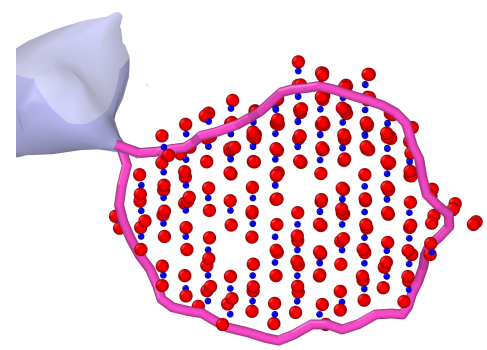

(e) $4.00 \mathrm{ps}$

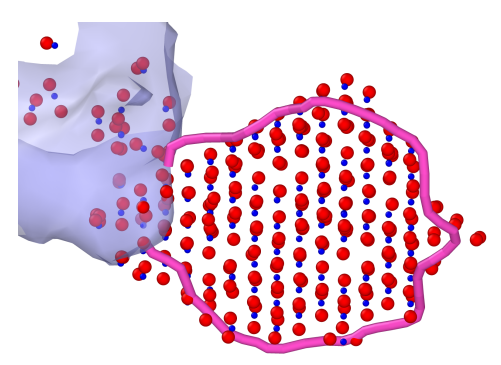

(c) $1.71 \mathrm{ps}$

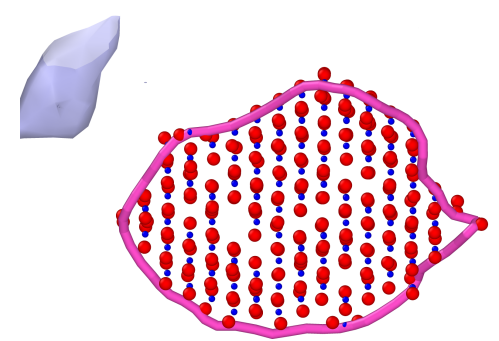

(f) $30.00 \mathrm{ps}$

FIG. 4: Destruction of the $1 / 2\langle 111\rangle$ segment of a mixed loop, and transformation into a perfect $\langle 100\rangle$ loop. The large (online: red) dots are the actual interstitial atoms and the small (online: blue) dots are the positions of the interstitial atom according to Wigner-Seitz analysis. The colouring is the same as for Fig. 1

loops, the processes leading to the larger $\langle 100\rangle$ loops also always entailed a transformation of the dislocation network within the (re)crystallized material. Hence, the stabilizing effect of e.g. $\mathrm{He}^{12}$ on interstitial clusters will likely inhibit this mechanism, in line with the findings that the $\langle 100\rangle$ loop population is significantly reduced in the presence of $\mathrm{He}^{22}$. On the other hand, our simulations show that the presence of $\mathrm{Cr}$ does not inhibit this process, as the $\langle 100\rangle$ loops were formed at all chromium contents, which is also in line with the experimental results reported in Ref. 22, where significant numbers of $\langle 100\rangle$ loops are seen also in irradiated FeCr samples.

Experimentally, TEM investigations on thin samples show that a large fraction of interstitial $\langle 100\rangle$ loops are formed at elevated temperatures ${ }^{34}$. At lower temperatures, vacancy type $\langle 100\rangle$ loops have been observed in thin film samples ${ }^{11,34}$, where supersaturation of vacancies is present. In thin film experiments, the time scale for cascade overlap competes with the time for loss of mobile interstitial defects to the surface, leading to reduced cascade overlap 
effects as compared to bulk irradiation, and hence a reduced probability for formation of $\langle 100\rangle$ interstitial-type loops at a given dose rate via this mechanism.

In summary, using molecular dynamics simulations, we have shown that $\langle 100\rangle$ interstitial dislocation loops can form directly as a result of overlapping cascades in Fe and FeCr. The process is cascade-induced, and takes place over picosecond time scales. We see that the energetic overlapping cascades can induce the transformation and combination of irregular $1 / 2\langle 111\rangle$ loops into $\langle 100\rangle$ loops, with no restrictions of initial size and configuration on the $1 / 2\langle 111\rangle$ loops. Furthermore, we observe transformations from various complex mixed $1 / 2\langle 111\rangle-\langle 100\rangle$ dislocation networks into complete $\langle 100\rangle$ loops through a sequence of $1 / 2\langle 111\rangle$ segments combining into $\langle 100\rangle$ segments. The results also show that the presence of debris from previous cascades is sufficient for the spontaneous in-cascade formation of small $\langle 100\rangle$ loops, even at low recoil energies.

\section{ACKNOWLEDGMENTS}

This work was partially funded by the Academy of Finland SIRDAME project. This work has been carried out within the framework of the EUROfusion Consortium and has received funding from the Euratom research and training programme 2014 - 2018 under grant agreement No 633053. The views and opinions expressed herein do not necessarily reflect those of the European Commission. Grants of computer time from the Center for Scientific Computing in Espoo, Finland, are gratefully acknowledged.

* fredric.granberg@helsinki.fi

1 Aliaga, M. J., Schäublin, R., LÖffler, J. F., and Caturla, M. J. Surface-induced vacancy loops and damage dispersion in irradiated fe thin films. Acta Mater. 101 (2015), 22 30.

2 Arakawa, K., Hatanaka, M., Kuramoto, E., Ono, K., and Mori, H. Changes in the Burgers Vector of Perfect Dislocation Loops without Contact with the External Dislocations. Phys. Rev. Lett. 96, 12 (Mar. 2006), 125506.

3 Béland, L. K., Osetsky, Y. N., Stoller, R. E., And Xu, H. Interstitial loop transformations in FeCr. J. Alloys Compd. 640 (Aug. 2015), 219-225. 
4 Calder, A. F., Bacon, D. J., Barashev, A. V., And Osetsky, Y. N. Effect of mass of the primary knock-on atom on displacement cascade debris in $\alpha$-iron. Phil. Mag. Lett. 88, 1 (Jan. 2008), 43-53.

5 Callister, JR., W. D. Materials Science and Engineering, An Introduction, third ed. Wiley, New York, 1993.

6 Dudarev, S. L., Bullough, R., And Derlet, P. M. Effect of the $\alpha-\gamma$ Phase Transition on the Stability of Dislocation Loops in bcc Iron. Phys. Rev. Lett. 100, 13 (Apr. 2008), 135503.

7 Dudarev, S. L., And Derlet, P. A 'magnetic' interatomic potential for molecular dynamics simulations. J. Phys. Condens. Matter 17 (2005), 1.

8 Dudarev, S. L., Gilbert, M. R., Arakawa, K., Mori, H., YaO, Z., Jenkins, M. L., And Derlet, P. M. Langevin model for real-time Brownian dynamics of interacting nanodefects in irradiated metals. Phys. Rev. B 81 (Jun 2010), 224107.

9 Ghaly, M., Nordlund, K., And Averback, R. S. Molecular dynamics investigations of surface damage produced by kiloelectronvolt self-bombardment of solids. Phil. Mag. A 79, 4 (1999), 795 .

10 Henriksson, K. O. E., Buörkas, C., And Nordlund, K. Atomistic simulations of stainless steels: a many-body potential for the Fe-Cr-C system. J. Phys. Condens. Matter 25, 44 (2013), 445401.

11 Jenkins, M. L., English, C. A., And Eyre, B. L. Heavy-ion irradiation of $\alpha$-iron. Phil. Mag. A 38, 1 (1978), 97-114.

12 Lucas, G., And Schäublin, R. Helium effects on displacement cascades in $\alpha$-iron. J. Phys. Condens. Matter 20, 41 (2008), 415206.

13 Malerba, L., Marinica, M., Anento, N., Buörkas, C., Nguyen, H., Domain, C., Duurabekova, F., Olsson, P., Nordlund, K., Serra, A., Terentyev, D., Willaime, F., And Becquart, C. Comparison of empirical interatomic potentials for iron applied to radiation damage studies. J. Nucl. Mater. 406, 1 (2010), 19 - 38.

14 Marian, J., Wirth, B. D., And Perlado, J. M. Mechanism of Formation and Growth of $\langle 100\rangle$ Interstitial Loops in Ferritic Materials. Phys. Rev. Lett. 88, 25 (June 2002), 255507.

15 Marinica, M.-C., Willaime, F., And Crocombette, J.-P. Irradiation-induced formation of nanocrystallites with $c 15$ laves phase structure in bcc iron. Phys. Rev. Lett. 108 (Jan 2012), 025501. 
16 Masters, B. C. Dislocation loops in irradiated iron. Phil. Mag. 11, 113 (May 1965), 881-893.

17 Mendelev, M. I., Han, S., Srolovitz, D. J., Ackland, G. J., Sun, D. Y., And Asta, M. Development of new interatomic potentials appropriate for crystalline and liquid iron. Phil. Mag. 83, 35 (2003), 3977-3994.

18 Nordlund, K., And Averback, R. S. Point defect movement and annealing in collision cascades. Phys. Rev. B 56, 5 (1997), 2421-2431.

19 Nordlund, K., Ghaly, M., Averback, R. S., Caturla, M., Diaz de la Rubia, T., AND TARus, J. Defect production in collision cascades in elemental semiconductors and FCC metals. Phys. Rev. B 57, 13 (1998), 7556-7570.

20 Norgett, M. J., Robinson, M. T., And Torrens, I. M. A proposed method of calculating displacement dose rates. Nucl. Eng. Design 33, 1 (1975), 50-54.

21 Olsson, P., Wallenius, J., Domain, C., Nordlund, K., and Malerba, L. Two-band modeling of alpha-prime phase formation in Fe-Cr. Phys. Rev. B 72, 21 (Dec. 2005), 214119.

22 Prokhodtseva, A., Décamps, B., Ramar, A., And Schäublin, R. Impact of he and cr on defect accumulation in ion-irradiated ultrahigh-purity fe(cr) alloys. Acta Mater. 61 (2013), 6958-6971.

23 Rau, R. C., D'Aragona, F. S., And Ladd, R. L. Neutron damage in molybdenum irradiated at high temperatures. Phil. Mag. 21, 171 (Mar. 1970), 441-452.

24 Rice, P. M., And Zinkle, S. J. Temperature dependence of the radiation damage microstructure in V-4cr-4ti neutron irradiated to low dose. J. Nucl. Mater. 258-263, Part 2 (Oct. 1998), $1414-1419$.

25 Sand, A. E., Dudarev, S. L., And Nordlund, K. High-energy collision cascades in tungsten: Dislocation loops structure and clustering scaling laws. EPL 103, 4 (2013), 46003.

26 Soneda, N., Ishino, S., And de la Rubia, T. D. Vacancy loop formation by 'cascade collapse' in a-fe: A molecular dynamics study of 50kev cascades. Phil. Mag. Lett. 81, 9 (2001), 649-659.

27 Stoller, R. 1.11 - primary radiation damage formation. In Comprehensive Nuclear Materials, R. J. Konings, Ed. Elsevier, Oxford, 2012, pp. 293 - 332.

28 Stoller, R., Odette, G., And Wirth, B. Primary damage formation in bcc iron. J. Nucl. Mater. 251 (1997), $49-60$. 
29 Stukowski, A. Visualization and analysis of atomistic simulation data with OVITO - the Open Visualization Tool. Model. Simul. Mater. Sci. Eng. 18, 1 (2010), 015012.

30 Stukowski, A., Bulatov, V. V., And Arsenlis, A. Automated identification and indexing of dislocations in crystal interfaces. Model. Simul. Mater. Sci. Eng. 20, 8 (2012), 085007.

31 Terentyev, D., Vörtler, K., Buörkas, C., Nordlund, K., And Malerba, L. Primary radiation damage in bcc Fe and Fe-Cr crystals containing dislocation loops. J. Nucl. Mater. 417, 1-3 (Oct. 2011), 1063-1066.

32 Willaime, F., Fu, C. C., Marinica, M. C., and Dalla Torre, J. Stability and mobility of self-interstitials and small interstitial clusters in $\alpha$-iron: ab initio and empirical potential calculations. Nucl. Inst. Meth. Phys. Res. B 228 (Jan. 2005), 92-99.

33 Xu, H., Stoller, R. E., Osetsky, Y. N., and Terentyev, D. Solving the Puzzle of $\langle 100\rangle$ Interstitial Loop Formation in bcc Iron. Phys. Rev. Lett. 110, 26 (June 2013), 265503.

34 Yao, Z., Jenkins, M. L., Hernández-Mayoral, M., and Kirk, M. A. The temperature dependence of heavy-ion damage in iron: A microstructural transition at elevated temperatures. Phil. Mag. 90, 35-36 (Dec. 2010), 4623-4634.

35 Yi, X., Jenkins, M. L., Briceno, M., Roberts, S. G., Zhou, Z., and Kirk, M. A. In situ study of self-ion irradiation damage in $\mathrm{W}$ and $\mathrm{W}-5$ Re at $500{ }^{\circ} \mathrm{C}$. Phil. Mag. 93, 14 (May 2013), 1715-1738.

36 Zhang, S., Nordlund, K., Duurabekova, F., Granberg, F., Zhang, Y., and Wang, T. S. Radiation damage buildup by athermal defect reactions in nickel and concentrated nickel alloys. Mater. Res. Lett. 5, 6 (2017), 433-439.

37 Zhang, Y., Bai, X. M., Tonks, M. R., And Biner, S. B. Formation of prismatic loops from C15 Laves phase interstitial clusters in body-centered cubic iron. Scripta Mater. 98 (Mar. 2015), 5-8. 